\title{
Análisis espacial con R: Usa R como un Sistema de Información Geográfica
}

Jean-François, Mas, European Scientific Institute, 2018, 151 pp. ISBN: 978-608-464266-4. Disponible en http://eujournal.org/files/journals/1/books/JeanFrancoisMas.pdf

\section{Elena, Chicaiza Mora R-Ladies, Quito, Ecuador}

El libro titulado Análisis espacial con R: Usa $R$ como un Sistema de Información Geográfica, realiza una breve introducción de los principales conceptos para el manejo del lenguaje y entorno de programación $\mathrm{R}$ (tipos de objetos y operaciones básicas) para posteriormente acercar al lector al uso de la librería o paquete $s f$, para datos espaciales en formato vector a través de sus principales funciones para lectura, escritura y análisis. Y la librería raster para datos en formato raster.

El libro aborda, desde una perspectiva práctica y aplicativa con un lenguaje de fácil entendimiento, los primeros pasos para iniciar con el manejo de $\mathrm{R}$ en aplicaciones de análisis espacial; para ello, es necesario que los usuarios tengan conocimientos básicos de Sistemas de Información Geográfica, es un material de referencia en español para quienes no están familiarizados con la programación en $\mathrm{R}$.

En los primeros capítulos, el autor de una forma sencilla, explica el funcionamiento de $\mathrm{R}$ a través de la instalación, versiones, paquetes y la interface más popular, como es R-Studio, para luego hacer una clara descripción de la estructura de los datos como objetos y objetos espaciales en $\mathrm{R}$, así como sus principales operaciones básicas.

La organización de los objetos espaciales en $\mathrm{R}$, y su representación espacial es abordada desde los dos modelos de datos geográficos básicos como son: vector y raster, con el uso de los paquetes $s f$ y raster respectivamente. Es importante destacar la recomendación del uso del paquete $s f$, y no el paquete $s p$ que fue el primero y más difundido. Esta recomendación se debe al lanzamiento del paquete $s f$ en el año 2017, que presenta una estructura de los datos más simple y poco a poco desplaza el uso del paquete $s p$.

Con respecto a la importanción y exportación de datos espaciales, se presentan los fomatos más conocidos tanto para vector, como es el caso del formato shape, y 
Geodatabase de ESRI y de raster con diferentes formatos de imagen y la opción de escoger la codificación de los datos.

En los capítulos 5 y 6 se presentan algunas operaciones básicas vectoriales, como: intersección, cálculo de distancias, áreas de influencia (buffers), agregación espacial. En el caso de las operaciones básicas raster que se abordan en el libro están: creación de mosaicos, recorte, reclasificación de valores, agregación espacial. Estas funciones se realizan a través de la librería raster. Adicionalmente, se muestran ejemplos de ejecución de métodos de re-muestreo (resample) como: vecino más cercano, bilineal; y operaciones de filtrado espacial. Por otro lado, se muestran operaciones de rasterización y de álgebra de mapas.

El capítulo 7, titulado "Análisis geoestadístico: detección de hot spots", describe el uso de la librería spdep para la definición y visualización de un atributo (ponderaciones espaciales). Se presenta un estudio de caso de estadística espacial, sin embargo no se aborda el tema de geoestadística propiamente, pues en $\mathrm{R}$ existen las librerías geor, geostat, Rgeostats.

El análisis de sensores remotos, es abordado en el capítulo 8, iniciando con la importación de un conjunto de bandas. La visualización y el pre-tratamiento, así como la posibilidad de realizar fusión entre bandas multiespectrales y pancromática. El cálculo de índices espectrales, como SAVI, NDVI también es mostrado.

Por su parte, la clasificación supervisada y no supervisada de imágenes satelitales se la pueda realizar a través de la aplicación de la librería raster. El libro describe brevemente otros paquetes con los cuales es posible procesar diferentes fuentes de sensores remotos, incluyendo LiDAR.

En el capítulo 9, se presentan funciones para la creación de mapas sencillos, principalmente usando las librerías: maptools y RColorBrewer. Y en el capítulo 10 se hace una breve reseña del uso de R con QGIS y Dinámica EGO.

Finalmente, en la sección de Anexos, se presenta un apartado en donde se aborda el uso de los conceptos y la lógica de la librería $s p$ para la gestión de datos espaciales, dado que esta librería ha sido usada extensivamente en la comunidad de usuarios de $\mathrm{R}$ en los últimos años.

De manera general, el libro presenta una estructura de fácil entendimiento para el manejo de datos espaciales con $\mathrm{R}$, desde un punto bastante operativo, sin centrarse en algoritmos y conceptos de análisis espacial, buscando siempre, permitir al lector no desanimarse en el aprendizaje del entorno de progración que tiene $\mathrm{R}$ y motivando la profundización del aprendizaje a través de las comunidades y redes colaborativas. 